\title{
Effect of an Acute Bout of Whole Body Vibration EXercise on Muscle Force OUTPUT AND Motor NeUron Excitability
}

\author{
Jeffrey M. McBride, James L. Nuzzo, Andrea M. Dayne, Michael A. Israetel, \\ David C. Nieman, and N. Travis Triplett \\ Neuromuscular Laboratory, Department of Health, Leisure, and Exercise Science, Appalachian State University, \\ Boone, North Carolina
}

\begin{abstract}
McBride, JM, Nuzzo, JL, Dayne, AM, Israetel, MA, Nieman, DC, and Triplett, NT. Effect of an acute bout of whole body vibration exercise on muscle force output and motor neuron excitability. J Strength Cond Res 24(1): 184-189, 2010-The purpose of the current investigation was to assess the effect of an acute bout of whole body vibration (WBV) exercise on muscle force output and motor neuron excitability. Nineteen recreationally trained college-aged males were randomly assigned to a WBV ( $n=10$ ) or a sham (S, $n=9$ ) group. The WBV group completed a series of static, body weight squats on a vibrating platform at $30 \mathrm{~Hz}$ and an amplitude of $\sim 3.5 \mathrm{~mm}$ (vertical), whereas the $S$ group performed the same series of exercises but without vibration. Measurements were performed before (Pre) and then immediately post-exercise (Imm Post), 8 minutes post-exercise (8-Min Post), or 16 minutes post-exercise (16-Min Post) during 3 different testing sessions. The measurements involved a ballistic isometric maximum voluntary contraction (MVC) of the triceps surae muscle complex and electrical stimulation of the tibial nerve for assessment of motor neuron excitability by analyzing $\mathrm{H}$-reflex and $\mathrm{M}$-wave responses $\left(H_{\max } / M_{\max }\right.$ ratio). Electromyography was also obtained from the triceps surae muscle complex during the MVCs. The WBV group significantly ( $p \leq 0.05$ ) increased peak force at Imm Post (9.4\%) and 8-Min Post (10.4\%). No significant change in peak force was observed in the $\mathrm{S}$ group. No significant changes were observed in either group for average integrated EMG, $H_{\max } / M_{\max }$ ratio, or rate of force development at Imm Post, 8-Min Post, or 16-Min Post. The results from this investigation indicate that an acute bout of static, body weight squat exercises, combined with WBV, increases muscle force output up to 8 minutes
\end{abstract}

Address correspondence to Jeffrey M. McBride, mcbridejm@appstate. edu.

24(1)/184-189

Journal of Strength and Conditioning Research

(C) 2010 National Strength and Conditioning Association post-exercise. However, this increase in muscle force is not accompanied by a significant increase in motor neuron excitability or muscle activation. Thus, it is plausible to use WBV as a method for acute increase in muscle force output for athletes immediately before competition.

KEY WoRDS potentiation, electromyography, $\mathrm{H}$ reflex, $\mathrm{M}$ wave

\section{INTRODUCTION}

$\mathrm{T}$ he use of whole body vibration (WBV) as an exercise modality is relevant to sports scientists and strength and conditioning coaches due to the array of physiological and performance measurements which it affects (2-5). In particular, acute exposure to WBV has been shown to result in increased respiration $(19,20)$ and energy expenditure (9), increased cutaneous blood flow (19), increased anabolic hormone response (12), and increased activation of the tonic vibration reflex (16). However, the use of an acute bout of WBV as a method to potentiate athletic performance measures remains unclear $(3,6,8)$.

Acute WBV has been found to cause both improvements and decrements in performance $(3,8)$. These conflicting observations appear to be the result of the different frequencies and amplitudes of the vibration utilized, the mode of the exercise prescribed, the outcome variables measured, and the post-treatment time point when the measurement were taken (25). Two studies have demonstrated that acute WBV has a potentiating effect on vertical jump performance $(5,8)$. Cormie et al. (8) discovered that a single set of static squats for 30 seconds with WBV $(30 \mathrm{~Hz}$, peak-to-peak amplitude of $2.5 \mathrm{~mm}$ ) caused a significant increase in vertical jump height measured immediately post-exercise (Imm Post) when compared with a sham group (S). Furthermore, using a more extensive exercise protocol ( 10 sets of static squats held for 60 seconds per set with the addition of WBV $[26 \mathrm{~Hz}$, amplitude of $4 \mathrm{~mm}]$ ), Bosco et al. (5) discovered significant improvements in both vertical jump height and power. However, not all research supports the use of acute WBV for improved performance $(8,10)$. Cormie et al. $(8)$ observed no significant differences between vibration and sham groups when

184 Journal of Strength and Conditioning Research 
Journal of Strength and Conditioning Research" $\mid$ www.nsca-jscr.org

comparing isometric squat peak force, vertical jump peak power, and muscle activation from Imm Post up until 30 minutes post-treatment. Additionally, de Ruiter et al. (10) discovered that 5 sets of 1 minute of static squats with WBV $(30 \mathrm{~Hz}$, amplitude of $8 \mathrm{~mm}$ ) resulted in significant reductions in muscle force output and muscle activation from 90 seconds post-vibration up until 180 minutes post-vibration. Furthermore, when using an exhaustive exercise protocol (squats with additional loads of $40 \%$ of body weight) in conjunction with WBV $(26 \mathrm{~Hz}$, amplitude of $1.05 \mathrm{~cm})$, a significant reduction in vertical jump height has been observed (24). Thus, due to the conflicting data reported regarding the effects of acute WBV on performance, it appears that further research on this topic is warranted.

Unfortunately, the physiological mechanisms by which these varied results in performance have occurred are currently not well understood. It seems plausible that performance after acute WBV would be reliant on the relationship between the levels of fatigue and postactivation potentiation (PAP) caused by the acute exposure. PAP is a condition in which pre-exercise muscle contractions lead to subsequent enhanced muscle force output through either increased motor neuron excitability or phosphorylation of myosin light chains $(13,17,22,26)$. Motor neuron excitability has been measured indirectly by utilizing a technique involving tibial nerve stimulation in many investigations $(3,11,15,18,21,27)$. Two electromyographic wave patterns appear during this technique. The $\mathrm{M}$ wave is a single twitch of the electrically stimulated muscle, and the $\mathrm{H}$ reflex is a wave pattern resulting from activation of a monosynaptic reflex response as a result of electrical stimulation to the same muscle (18). Expressing the H-reflex wave amplitude with respect to the $\mathrm{M}$-wave amplitude allows for a ratio indicative of the motor neuron excitability (18). The effect of WBV on $\mathrm{H}$ reflex has been studied previously (3). Armstrong et al. (3) discovered that the H-reflex response was significantly reduced in all subjects after 1 minute of WBV $(40 \mathrm{~Hz}$, amplitude of $2-4 \mathrm{~mm}$ ); however, the response after 1 minute varied greatly among subjects as some recovered to baseline levels as early as 3 minutes post-vibration, whereas others did not recover even at 27 minutes post-vibration.

To date, there appears to be a paucity of research attempting to provide concurrent assessments of both muscle force output and motor neuron excitability after an acute bout of WBV. Therefore, the purpose of this investigation was to determine the effects of acute WBV exercise on muscle force output, rate of force development (RFD), and motor neuron excitability. Thus, this study may help determine whether WBV may be beneficial as a warm-up procedure before athletic activity, which is contingent upon muscle force output.

\section{Methods}

\section{Experimental Approach to the Problem}

This study investigated the effects of acute exposure to WBV on muscle force output and nervous system function. Subjects were exposed to either a series of exercises without WBV or with WBV. Maximal force production, rate of force development, average integrated EMG, and $\mathrm{H}_{\max } / \mathrm{M}_{\max }$ ratios were measured to assess acute changes in neuromuscular function.

\section{Subjects}

Nineteen recreationally trained subjects participated in this study. The subjects were partitioned into experimental (WBV, $n=10)($ age $=22.6 \pm 2.2$ years; body height $=175.9 \pm$ $6.5 \mathrm{~cm}$; body mass $=77.5 \pm 10.7 \mathrm{~kg})$ and sham $(\mathrm{S}, n=9)$ $($ age $=20.8 \pm 1.2$ years; body height $=172.5 \pm 11.4 \mathrm{~cm}$; body mass $=83.2 \pm 11.1 \mathrm{~kg}$ ) groups. There were no statistically significant differences between the groups for the above variables. All subjects were males aged between 18 and 27 years who had been participating in resistance and/or aerobic training regimens over the past 2 years. Subjects were asked to refrain from their current training practices 48 hours before each testing session to avoid any effect of traininginduced fatigue on performance variables and motor neuron excitability. All subjects signed a consent form, which along with the study protocol was approved by the Institutional Review Board at Appalachian State University.

\section{Study Design}

Each subject participated in 4 separate testing sessions with each session separated by at least 24 hours but no more than 72 hours. The subjects were randomly assigned into the experimental (WBV) and S groups. The WBV received the vibration treatment during their treatment protocol, and the $S$ group performed an identical protocol on the same vibration platform, except that no vibrations were administered. The first session served as a familiarization in which subjects completed 3 maximal voluntary contractions (MVCs) of the triceps surae complex and also the treatment protocol exercises. The next 3 sessions involved pre-treatment (Pre) and post-treatment measurements of motor neuron excitability, and peak force, RFD, and soleus muscle average integrated electromyography (IEMG) during an MVC. Posttreatment measurements occurred Imm Post, 8 minutes post-treatment (8-Min Post), or 16 minutes post-treatment (16-Min Post) during the 3 different testing sessions (Figure 1). Three separate testing sessions were utilized to prevent any confounding effect of non-treatment factors (i.e., posttreatment MVCs) on the study outcome measures at those time points. The order in which the 3 sessions were completed was randomized.

\section{Treatment Protocol}

The WBV and $\mathrm{S}$ groups performed identical protocols (Table 1) on a vibration platform (Power Plate International, Irvine, CA, USA); however, only WBV received the vibration treatment. Six sets of static, body weight squats were performed. The first 3 sets were performed bilaterally for 30 seconds at a frequency of $30 \mathrm{~Hz}$ and at the high-amplitude (approximately $3.5 \mathrm{~mm}$ ) setting. Thereafter, subjects completed 


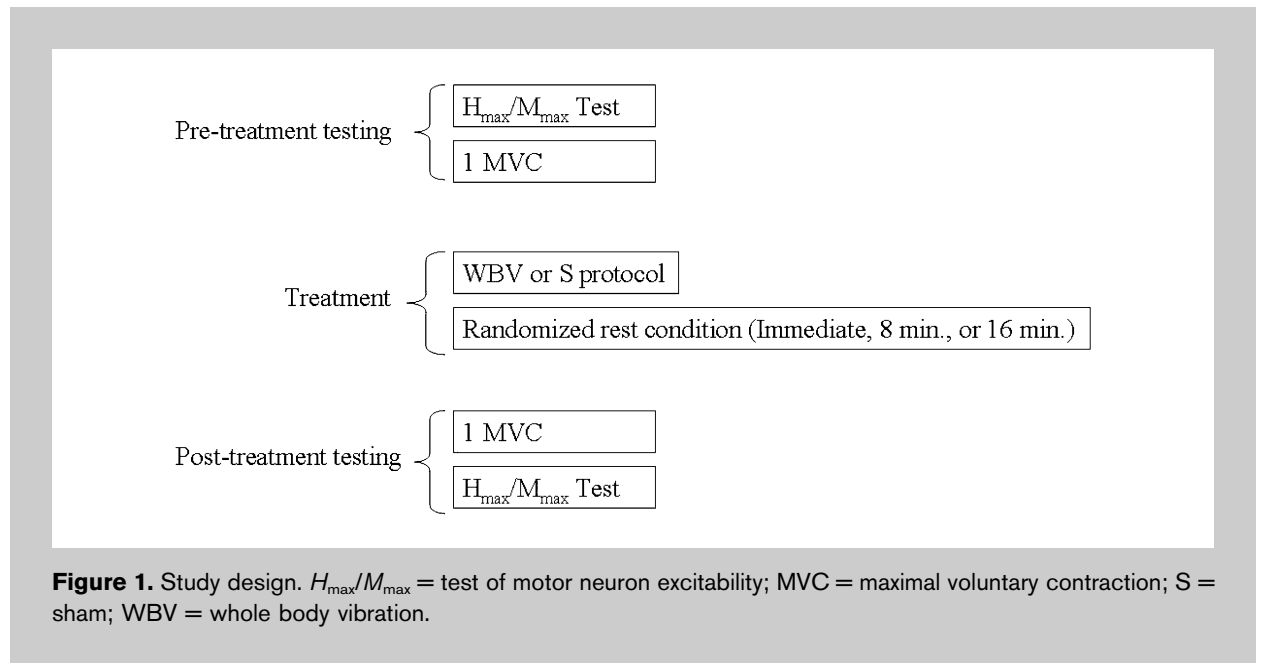

3 sets of unilateral static squats (3 with the right leg and 3 with the left leg in an alternating fashion). Subjects completed 30 seconds of the exercise with the right leg and then immediately completed 30 seconds on the left leg, and this was considered 1 set. A 60 -second seated rest period was provided between all sets. All squats were held at a $100^{\circ}$ knee angle as measured by a goniometer, and subjects were required to grasp the handles, but instructed not to pull or push using their arm, on the vibration platform while completing the exercises.

\section{Maximal Voluntary Contractions and Rate of Force Development}

Maximal voluntary contractions of the triceps surae complex occurred in a seated position utilizing a force plate (BP6001200; AMTI, Watertown, MA) and an isometric rack (Figure 2). Subjects were positioned so that $90^{\circ}$ joint angles were attained at the hip, knee, and ankle. The feet, without shoes, were placed on a custom-designed platform, which was located directly on top of a force plate. An isometric rack bar was positioned on top of the subject's distal thigh at a point approximately $5 \mathrm{~cm}$ from the superior portion of the patella. The location of the bar on the thigh was then marked using permanent marker to replicate the same bar position for every trial. Additionally, the position of the feet on the platform was marked to replicate positioning for each trial. A foam pad was placed around the bar to eliminate discomfort during the MVCs and to ensure that maximal efforts were obtained. To compensate for differences in segment lengths and body heights between subjects, the bar position could be moved vertically with the use of hydraulic pumps. One MVC was performed before the treatment, and one MVC was performed after the treatment. Single MVC trials were utilized to prevent any confounding effect of additional MVC trials on fatigue and PAP. All MVCs were 3 seconds in duration, and for all trials, subjects were instructed to develop maximal force as quickly as possible. A custom-designed LabVIEW (Version 8.2; National Instruments, Austin, TX) program was used to evaluate peak force and RFD based on force-time curves. Peak force was determined as the maximal force achieved, and RFD was determined as the change in force from the start of the MVC to the force at 200 milliseconds and then divided by 200 milliseconds.

\section{Motor Neuron Excitability and Electromyography}

Motor neuron excitability was assessed using percutaneous stimulation of the tibial nerve. Before pre-treatment measurements, the areas of the popliteal fossa and soleus muscle of the left leg were shaved, abraded, and swabbed with alcohol. The receiving electrode (Noraxon USA, Inc., Scottsdale, AZ; 2 -cm interelectrode distance, $1 \mathrm{~cm}^{2}$ circular conductive area) on the soleus muscle was positioned approximately $13 \mathrm{~cm}$ above the superior portion of the calcaneus and below the muscle fibers of the gastrocnemius (1). A remote stimulus device (Nicolet Viking II; Nicolet Biomedical, Madison, WI) was then used to locate the optimal site for stimulation of the tibial nerve (18). After the optimal site was determined, a bipolar stimulating electrode $(1-\mathrm{cm}$ conduction area and $3-\mathrm{cm}$ interelectrode distance) was secured in the same area using adhesive tape. The motor neuron excitability test was completed with subjects in a seated position with $90^{\circ}$ joint

TABLE 1. Treatment protocol.*

\begin{tabular}{ccccc}
\hline Exercise & Sets & Duration $(\mathrm{s})$ & Rest $(\mathrm{s})$ & Frequency $(\mathrm{Hz})$ \\
\hline Bilateral static squat $\left(100^{\circ}\right)$ & 3 & 30 & 60 & 30 \\
Unilateral static squat $\left(100^{\circ}\right)$ & 3 & 30 (each leg) & 60 & 30 \\
\hline
\end{tabular}

${ }^{\star}$ Total time (including rest between sets) $=8.5$ minutes; total time of exercise treatment $=4.5$ minutes. 


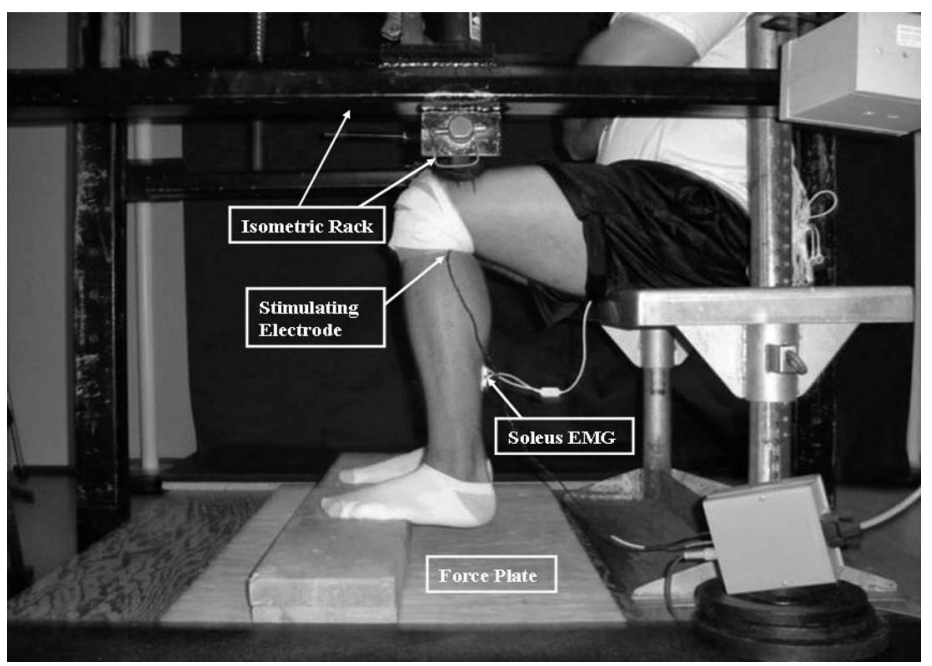

Figure 2. Maximal voluntary contraction and nerve stimulation testing setup.

to-digital converter; Noraxon USA, Inc., Scottsdale, AZ). The amplified myoelectric signal was detected by a receiveramplifier (Telemyo 900, gain $=$ 2000 , differential input impedance $=10 \mathrm{M} \Omega$, bandwidth frequency $=10-500 \mathrm{~Hz}$, common mode rejection ratio $=85$ dB; Noraxon USA, Inc.) and then sent to an $\mathrm{A} / \mathrm{D}$ card (NI PCI-6014; National Instruments) at $1000 \mathrm{~Hz}$. The signal was full-wave rectified and filtered (6-pole Butterworth notch filter $60 \mathrm{~Hz}$, band pass filter $10-200 \mathrm{~Hz})$. The integrated value $(\mathrm{mV} \cdot \mathrm{s})$ was calculated and then averaged to determine IEMG $(\mathrm{mV})$ for the entire 3 second MVC. Customdesigned programs written in

angles at the hip, knee, and ankle. The stimuli were delivered as 1-millisecond square pulses without simultaneous contraction of the triceps surae complex. Starting at a stimulus of $3 \mathrm{~V}$, the stimuli were administered in $3-\mathrm{V}$ increments at a rate of $0.1 \mathrm{~Hz}$ (18) until no further visual increase in the peak-to-peak M-wave amplitude was detected $(1,18)$. The stimulations elicited both $\mathrm{H}$-wave and $\mathrm{M}$-wave responses in the soleus muscle. The maximal $\mathrm{H}$ wave (i.e., $H_{\max }$ ) was expressed relative to the maximal $\mathrm{M}$ wave (i.e., $M_{\max }$ ) as the measure of motor neuron excitability $\left(H_{\max } / M_{\max }\right.$ ratio). During the MVCs, IEMG from the soleus muscle was measured. The myoelectric signal was collected through the use of a telemetry transmitter (8 channel, 12-bit analog-
LabVIEW (Version 8.2; National Instruments) were used for recording and analyzing the soleus EMG data.

\section{Statistical Analyses}

Test-retest reliability for MVC peak force and RFD were assessed using Cronbach alpha. A repeated-measures general linear model was utilized for comparisons of the outcome measures across the different testing sessions. An alpha level of 0.05 was chosen for all comparisons.

\section{Results}

The Pre values provided in Figures 3, 4, 5, and 6 represent the average Pre value for all the sessions for visual representation
only. Statistics were performed only. Statistics were performed on corresponding Pre values for each individual exercise session. A significant increase $(p \leq 0.05)$ in peak force during the MVC was observed Imm Post (9.4\%) and 8-Min Post (10.4\%) in the WBV group only (Figure 3 ). No significant changes in peak force were observed in the $S$ group. No significant changes in RFD were observed in either the WBV or S group (Figure 4). Between-trial reliability for MVC peak force and RFD data from the familiarization session was $r=0.93$ and $r=0.84$, respectively. Between-session reliability for MVC peak force and RFD was $r=0.86$ and $r=$
Figure 3. Peak force during the maximal voluntary contraction (MVC) before (Pre) and at immediate post-exercise (Imm Post), 8 minutes post-exercise (8-Min Post), and 16 minutes post-exercise (16-Min Post). *Significantly different from Pre $(p \leq 0.05)$. 


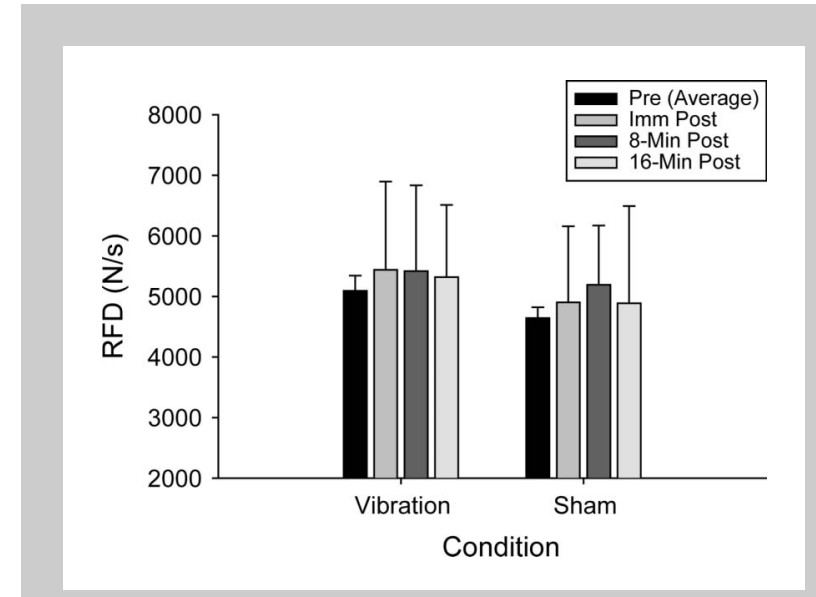

Figure 4. Rate of force development (RFD) during the maximal voluntary contraction (MVC) before (Pre) and at immediate post-exercise (Imm Post), 8 minutes post-exercise (8-Min Post), and 16 minutes postexercise (16-Min Post).

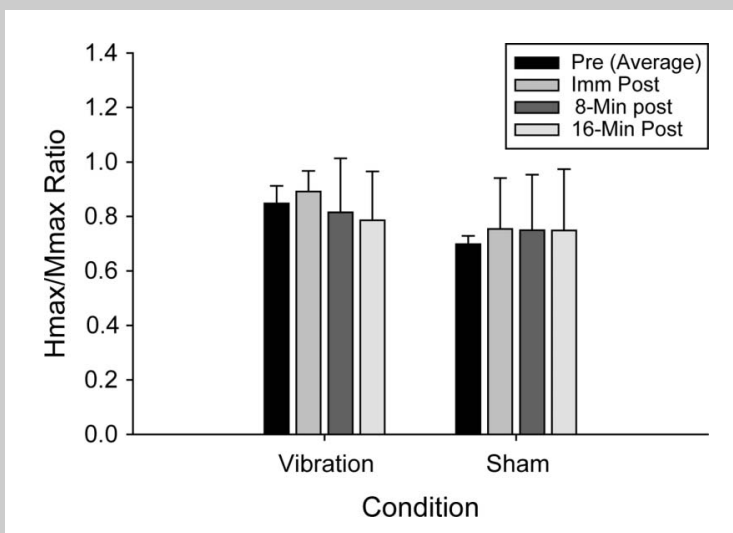

Figure 5. The $H_{\max } / M_{\max }$ ratio measured before (Pre) and at immediate post-exercise (Imm Post), 8 minutes post-exercise (8-Min Post), and 16 minutes post-exercise (16-Min Post).

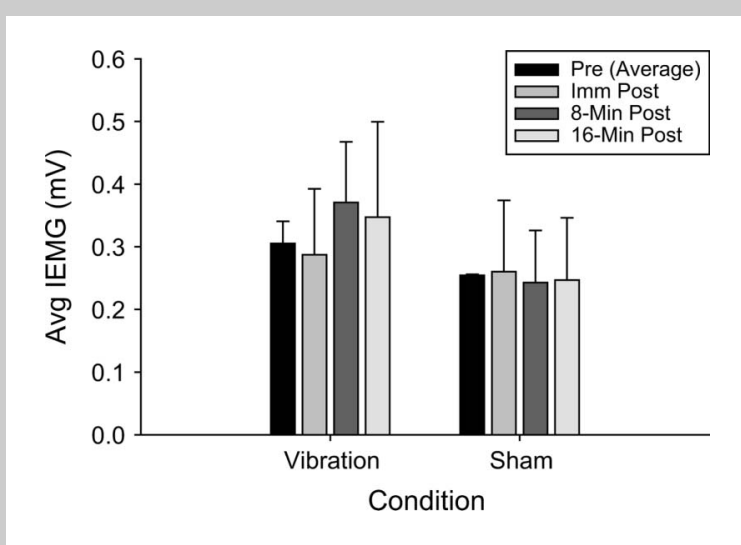

Figure 6. The average integrated electromyography (Avg IEMG) measured before (Pre) and at immediate post-exercise (Imm Post), 8 minutes post-exercise (8-Min Post), and 16 minutes post-exercise (16-Min Post).
0.83 , respectively. No significant changes were observed in $H_{\max } / M_{\max }$ ratio or muscle activity (IEMG) in either the WBV or S group (Figures 5 and 6).

\section{Discussion}

The findings from this investigation indicate that WBV combined with static, body weight squat exercises is sufficient to induce PAP as indicated by a significant increase in MVC peak force Imm Post and 8-Min Post. However, the mechanism for this increase in peak force is unclear as no changes in muscle activation motor neuron excitability were observed. It should be noted, however, that the statistical power for the motor neuron excitability measures was 0.69 and thus may have had a slightly negative effect on our ability to detect a meaningful change in this variable $\left(H_{\max } / M_{\max }\right.$ ratio). No previous studies have examined PAP as a result of WBV exercise and motor neuron excitability concurrently.

The results of the current investigation are in contrast to those of both Cormie et al. (8) and de Ruiter et al. (10). As stated previously, Cormie et al. (8) observed no significant differences between vibration and sham groups when comparing isometric squat peak force from Imm Post up until 30 minutes post-treatment. Additionally, de Ruiter et al. (10) discovered that 5 sets of 1 minute of static squats with WBV $(30 \mathrm{~Hz}$, amplitude of $8 \mathrm{~mm})$ resulted in significant reductions in muscle force output and muscle activation from 90 seconds post-vibration up until 180 minutes postvibration. Furthermore, when using an exhaustive exercise protocol (squats with additional loads of $40 \%$ of body weight) in conjunction with WBV $(26 \mathrm{~Hz}$, amplitude of $1.05 \mathrm{~cm})$, a significant reduction in vertical jump height has been observed (24). This protocol used in the current investigation may have been sufficient to induce PAP, yet conservative enough as to not induce a significant level of fatigue, thus masking the PAP effect on maximal muscle force output.

As previously discussed, several methods of inducing PAP have appeared in the literature $(14,17)$. However, limited information is available as to how WBV may affect this phenomenon. Due to the known activation of the tonic vibration reflex induced by WBV (16), it was theorized that the mechanism for PAP with WBV exercise might be an observable change in the $H_{\max } / M_{\max }$ ratio. The current study confirms that WBV can induce PAP, but the mechanism is unclear as the changes in the $H_{\max } / M_{\max }$ ratio were not statistically significant. It is possible that the level of motor neuron excitability was increasing but to a lesser extent and was undetectable by the nerve stimulation technique utilized in the current study due to a lower than optimal power level of 0.690 . However, muscle activation was also measured and did not change significantly. In addition, myosin light chain phosphorylation may have served as a mechanism for increasing muscle force output in the current investigation (13). However, because myosin light chain phosphorylation was not measured in the current investigation, its role in 
Journal of Strength and Conditioning Research" $\mid$ www.nsca-jscr.org

increasing muscle force output after an acute bout of WBV exercise is still unclear.

It is unclear why body weight exercise without vibration did not induce PAP, as it has been shown to occur in several past investigations $(7,23)$. However, the PAP response appears to be exercise intensity dependent (23) and may have been at an insufficient intensity in the current study. There appears to be a synergistic effect of body weight exercises performed in conjunction with WBV as observed by the significant increase in MVC peak force in the WBV group in the current investigation. Therefore, it appears that a combination of body weight exercise and WBV may be a practical way of improving athletic performance for up to 8Min Post if that athletic activity is dependent on maximal force production of the triceps surae complex.

\section{Practical Applications}

The current investigation indicates that $\mathrm{WBV}$ is effective for inducing a small degree of PAP. However, the physiological mechanisms responsible for this occurrence are still unclear. It should be noted that the level of PAP observed in this study could also be achieved by standardized warm-up procedures more commonly utilized by practitioners $(7,14)$. Therefore, WBV may be used as a viable tool for acutely increasing muscle force output immediately before competition.

\section{ACKNowledgment}

This study was supported by a grant from Power Plate International.

\section{REFERENCES}

1. Aagaard, P, Simonsen, EB, Andersen, JL, Magnusson, P, and DyhrePoulsen, P. Neural adaptation to resistance training: Changes in evoked V-wave and H-reflex responses. J Appl Physiol 92: 23092318, 2002.

2. Abercromby, AF, Amonette, WE, Layne, CS, McFarlin, BK, Hinman, MR, and Paloski, WH. Variation in neuromuscular responses during acute whole-body vibration exercise. Med Sci Sports Exerc 39: 1642-1650, 2007.

3. Armstrong, WJ, Nestle, HN, Grinnell, DC, Cole, LD, Van Gilder, EL, Warren, GS, and Capizzi, EA. The acute effect of whole-body vibration on the Hoffmann reflex. $J$ Strength Cond Res 22: 471-476, 2008.

4. Bogaerts, A, Delecluse, C, Claessens, AL, Coudyzer, W, Boonen, S, and Verschueren, SM. Impact of whole-body vibration training versus fitness training on muscle strength and muscle mass in older men: A 1-year randomized controlled trial. J Gerontol 62: 630-635, 2007.

5. Bosco, C, Iacovelli, M, Tsarpela, O, Cardinale, M, Bonifazi, M, Tihanyi, J, Viru, M, De Lorenzo, A, and Viru, A. Hormonal responses to whole-body vibration in men. Eur J Appl Physiol 81: 449-454, 2000.

6. Cardinale, M, Leiper, J, Erskine, J, Milroy, M, and Bell, S. The acute effects of different whole body vibration amplitudes on the endocrine system of young healthy men: A preliminary study. Clin Physiol Funct Imaging 26: 380-384, 2006.

7. Chiu, LZ, Fry, AC, Schilling, BK, Johnson, EJ, and Weiss, LW. Neuromuscular fatigue and potentiation following two successive high intensity resistance exercise sessions. Eur J Appl Physiol 92: 385-392, 2004
8. Cormie, P, Deane, RS, Triplett, NT, and McBride, JM. Acute effects of whole-body vibration on muscle activity, strength, and power. $J$ Strength Cond Res 20: 257-261, 2006.

9. Da Silva, ME, Fernandez, JM, Castillo, E, Nunez, VM, Vaamonde, DM, Poblador, MS, and Lancho, JL. Influence of vibration training on energy expenditure in active men. J Strength Cond Res 21: 470475, 2007.

10. de Ruiter, CJ, van der Linden, RM, van der Zijden, MJ, Hollander, AP, and de Haan, A. Short-term effects of whole-body vibration on maximal voluntary isometric knee extensor force and rate of force rise. Eur J Appl Physiol 88: 472-475, 2003.

11. Del Balso, $\mathrm{C}$ and Cafarelli, E. Adaptations in the activation of human skeletal muscle induced by short-term isometric resistance training. J Appl Physiol 103: 402-411, 2007.

12. Di Loreto, C, Ranchelli, A, Lucidi, P, Murdolo, G, Parlanti, N, De Cicco, A, Tsarpela, O, Annino, G, Bosco, C, Santeusanio, F, Bolli, GB, and De Feo, P. Effects of whole-body vibration exercise on the endocrine system of healthy men. $J$ Endocrinol Invest 27: 323-327, 2004.

13. Ebben, WP. A brief review of concurrent activation potentiation: Theoretical and practical constructs. J Strength Cond Res 20: 985991, 2006.

14. Gilbert, $G$ and Lees, A. Changes in the force development characteristics of muscle following repeated maximum force and power exercise. Ergonomics 48: 1576-1584, 2005.

15. Gruber, M, Taube, W, Gollhofer, A, Beck, S, Amtage, F, and Schubert, M. Training-specific adaptations of $\mathrm{H}$ - and stretch reflexes in human soleus muscle. J Mot Behav 39: 68-78, 2007.

16. Hagbarth, KE and Eklund, G. Tonic vibration reflexes (TVR) in spasticity. Brain Res 2: 201-203, 1966.

17. Hodgson, M, Docherty, D, and Robbins, D. Post-activation potentiation: Underlying physiology and implications for motor performance. Sports Med 35: 585-595, 2005.

18. Holtermann, A, Roeleveld, K, Engstrom, M, and Sand, T. Enhanced $\mathrm{H}$-reflex with resistance training is related to increased rate of force development. Eur J Appl Physiol 101: 301-312, 2007.

19. Hood, WB Jr, Murray, RH, Urschel, CW, Bowers, JA, and Clark, JG. Cardiopulmonary effects of whole-body vibration in man. $J$ Appl Physiol 21: 1725-1731, 1966.

20. Hoover, GN and Ashe, WF. Respiratory response to whole body vertical vibration. Aerosp Med 33: 980-984, 1962.

21. Laudani, L, Wood, L, Casabona, A, Giuffrida, R, and De Vito, G. Effects of repeated ankle plantar-flexions on $\mathrm{H}$-reflex and body sway during standing. J Electromyogr Kinesiol 19: 85-92, 2009.

22. Motl, RW, Knowles, BD, and Dishman, RK. Acute bouts of active and passive leg cycling attenuate the amplitude of the soleus $\mathrm{H}$-reflex in humans. Neurosci Lett 347: 69-72, 2003.

23. Paasuke, M, Rannama, L, Ereline, J, Gapeyeva, H, and Oopik, V. Changes in soleus motoneuron pool reflex excitability and surface EMG parameters during fatiguing low- vs. high-intensity isometric contractions. Electromyogr Clin Neurophysiol 47: 341-350, 2007.

24. Rittweger,J, Beller, G, and Felsenberg, D. Acute physiological effects of exhaustive whole-body vibration exercise in man. Clin Physiol 20: 134-142, 2000.

25. Ronnestad, BR. Comparing the performance-enhancing effects of squats on a vibration platform with conventional squats in recreationally resistance-trained men. J Strength Cond Res 18: 839$845,2004$.

26. Trimble, $\mathrm{MH}$ and Harp, SS. Postexercise potentiation of the H-reflex in humans. Med Sci Sports Exerc 30: 933-941, 1998.

27. Yamaguchi, T, Tanabe, S, Watanabe, T, and Muraoka, Y. Effect of voluntary contraction with electrical stimulation to antagonist muscle on agonist H-reflex. Electromyogr Clin Neurophysiol 47: 251255, 2007. 